\title{
Nonlinear Dynamic Analysis of Urban Roads and Daytime Population
}

\author{
Shiqing Yan \\ College of Urban and Planning, Yancheng Teachers University, Yancheng, China
}

\section{Email address:}

18360494968@163.com

\section{To cite this article:}

Shiqing Yan. Nonlinear Dynamic Analysis of Urban Roads and Daytime Population. Urban and Regional Planning. Vol. 2, No. 1, 2017, pp. 1-6. doi: 10.11648/j.urp.20170201.11

Received: February 13, 2017; Accepted: February 23, 2017; Published: March 9, 2017

\begin{abstract}
Since the road system can not meet the traffic demand generated by the daytime population, traffic congestion has restricted urban economic and social development. Based on nonlinear dynamics theory, this paper analyzes the relationship between the area of urban road and the daytime population. The result shows the number of urban daytime population is proportional to the area of urban road at steady state. The author also found that the increase of urban road area is constrained by the maximum road area of a particular area at the city. When the road area is fixed, the number of urban daytime population is inversely proportional to the travel intensity.
\end{abstract}

Keywords: Urban Road, Urban Daytime Population, Nonlinear Dynamics

\section{Introduction}

The daytime population refers to the people who are active in a particular area during the day, including people who are working, studying, shopping, travelling, visiting relatives and seeking medical treatment etc., and it also includes residential population who are not engage in economic activities. There is a one-day cycle of population migrates between the city center and the outskirts of the city. During the day, a large number of people from the city periphery to the central area of the city to engage in work, study, medical treatment, shopping, tourism and other activities, which dramatically increase the number of population in the city center. At night, the population flows in the opposite direction. The traffic demand arising from the population migration and the daytime population engaged in other activities, which is the main body of urban transport demand [1]. In the urban center area, the large density of daytime population and highly gathered people and traffic flow, this leads to traffic congestion [2]. Due to the small population density and inadequate traffic demand, it is difficult to develop public transportation and it is inconvenient to travel for residents [3]. Many scholars have explored the relationship between urban transport and urban population, and achieved a lot of research results [4-8]. However, they usually analyze the impact of urban population on urban transport in a general manner, and they ignore the effects of different components of urban daytime population on urban traffic.

Nonlinear dynamics theory can quantify the relationship between objects and evolutionary mechanism, and it is widely used to geographical sciences [9], environmental science [10], atmospheric sciences [11] and management science [12]. On the basis of a deep understanding of the intrinsic dynamics and the evolutionary mechanism of the objects, implement scientific and effective management, and then to predict, control and optimize the evolution process of the system. In this paper, the author proposes nonlinear theory to investigate the dynamical relationship between the urban road area and the daytime population, and predict the development tendency of the system which consisted of daytime population and urban road. It has important theoretical and practical significance for urban planning and urban management.

\section{Nonlinear Dynamics Model of Daytime Population and Urban Road Area}

According to the relationship between daytime population and urban road area, this paper proposed the following nonlinear dynamics model: 


$$
\left\{\begin{array}{l}
\frac{d x}{d t}=r x(a y-x) \\
\frac{d y}{d t}=R y\left[1-\left(A k_{1}+B k_{2}+C k_{3}\right) \frac{x}{y_{m}}\right]
\end{array}\right.
$$

where $x$ is the total number of daytime population in a city or an area, $y$ is the total area of road in that city or area. $r$ and $R$ are the growth rate of daytime population and road area, respectively. $a$ is the number of population that can be accommodated by the unit road area at normal condition, ay is the maximum number of population that can be accommodated by all of the roads in a city. $a y-x$ is the remaining capacity of population that can be accommodated by the urban road. The number 1 in the second equation of (1) is the restriction of regional resources, society and economy on urban road - urban daytime population system. The different components of a city's daytime population have different impacts on the urban economy and their transport demands are not the same. $k_{1}$ is the proportion of employed population that communicating into the city in urban daytime population. $A$ is the travel intensity of employed population that communicating into the city, and the travel intensity is effected by the number of trips, travel distance, travel time, and road area occupied by the trip. $k_{2}$ is the proportion of non-employed population that communicating into the city, including the population who commute into the city to go shopping, travel, medical treatment, visiting relatives and so on. $B$ is the travel intensity of non-employed population that communicating into the city. $k_{3}$ is the proportion of the residential population, $C$ is the travel intensity of the residential population. $y_{m}$ is the maximum road area determined by the regional resources, economic and social conditions. $\left(A k_{1}+B k_{2}+C k_{3}\right) x$ is the total traffic demand of urban daytime population. $1-\left(A k_{1}+B k_{2}+C k_{3}\right) \frac{x}{y_{m}}$ is the remaining capacity of urban road. Model (1) objectively reflects the constraint relations between the urban road area and the number of daytime population.

\section{The Solution and Discussion of Equilibrium State}

\subsection{The Solution of Equilibrium State}

Let $\frac{d x}{d t}=\frac{d y}{d t}=0$, we can get the equilibrium state solutions of model (1) are

$M(0,0)$ and

$$
N\left[x_{0}=\frac{y_{m}}{A k_{1}+B k_{2}+C k_{3}}, y_{0}=\frac{y_{m}}{a\left(A k_{1}+B k_{2}+C k_{3}\right)}\right] \text {. }
$$

\subsubsection{Equilibrium State $M(0,0)$}

According to the stability theory [13], it is observed that
$M(0,0)$ is an unstable saddle point, this implies that the system of urban road and urban daytime population will develop in saddle shaped and leave point $M(0,0)$.

\subsubsection{Equilibrium State}

$$
N\left[x_{0}=\frac{y_{m}}{A k_{1}+B k_{2}+C k_{3}}, y_{0}=\frac{y_{m}}{a\left(A k_{1}+B k_{2}+C k_{3}\right)}\right]
$$

Since $T=a_{11}+a_{22}=-r \frac{y_{m}}{A k_{1}+B k_{2}+C k_{3}}<0$, thus the equilibrium state $N$ is stable. According to the stability theory, when $r y_{m}-4 R\left(A k_{1}+B k_{2}+C k_{3}\right)>0$, the equilibrium state $N$ is a stable node, and its dynamic behavior is a non-periodic curve or linear motion, when $r y_{m}-4 R\left(A k_{1}+B k_{2}+C k_{3}\right)<0$, the equilibrium state $N$ is a stable focus, and its dynamic behavior is quasi-periodic motion with varying amplitude. Based on the above analysis, we derive the following conclusions:

(1) When $r>4 R\left(A k_{1}+B k_{2}+C k_{3}\right) / y_{m}$, that is the growth rate of daytime population is larger than four times of $R\left(A k_{1}+B k_{2}+C k_{3}\right) / y_{m}$, the system of urban road and daytime population transfer to equilibrium state $N$ with the tendency of non-periodic curve or linear motion and the final equilibrium state is stable. Because the movement speed of node is very fast, thus that is not benefit to the stability of the system. In reality, the growth speed of urban daytime population is too fast, and leads to a large number of economic and social problems.

(2) When $r<4 R\left(A k_{1}+B k_{2}+C k_{3}\right) / y_{m}$, that is the growth rate of daytime population is smaller than four times of $R\left(A k_{1}+B k_{2}+C k_{3}\right) / y_{m}$, the system evolves to equilibrium state $N$ in the way of quasi-periodic motion with varying amplitude, and the equilibrium state is stable. The quasi-periodic motion with varying amplitude implies the evolution of the system of urban road and daytime population is stable. In fact, the moderate increase of daytime population in the urban core area is the performance of economic development, industrial structure optimization and CBD functions increase. Therefore, it is meaningful to study the equilibrium state.

\subsection{Discussion of Equilibrium State}

Equilibrium state $N$ has important practical significance, and we will further discuss it. Equilibrium state $N$ shows (1) The number of urban daytime population $x$ is proportional to the maximum road area that a city can afford $y_{m}$, and inversely to the unit travel volume of daytime population $A k_{1}+B k_{2}+C k_{3}$. (2) The total area of urban roads $y$ is proportional to the maximum road area that a city can afford $y_{m}$, and inversely to the road through capacity $a\left(A k_{1}+B k_{2}+C k_{3}\right)$, where $a$ is the number of population that can be accommodated by the unit road area. (3) The number of daytime population is a times of road area at steady state. We discuss it in detail in the following. 


\subsubsection{Fix the Road Area, the Relationship Between the Number of Daytime Population $x$ and the Travel Volume $\boldsymbol{A k}_{1}+\boldsymbol{B k}_{2}+\boldsymbol{C k}_{3}$}

In the unit travel volume of daytime population $A k_{1}+B k_{2}+C k_{3}$, with the city CBD function, the number of employed population and non-employed population who go shopping, tourism and consulting into the city center are definitely increase, that means $k_{1}$ and $k_{2}$ are increase. Besides, with the improvement of quality of life, shopping and physical activity of residents will increase, and then the travel density of residential population is difficult to decrease. Therefore, in order to reduce the travel volume of unite daytime population, we need to take different strategies for different daytime population, and discuss their impacts on the number of daytime population $x$ respectively.

(1) Fix the road area, the relationship between the number of daytime population $x$ and the travel intensity of employed population $A$

When the road area is fixed, the increase of travel intensity of employed population that communicating into the city, which definitely leads to the increase of traffic demand, and then decrease the capacity of daytime population. We set $y_{m}$, $k_{1}$ and $B k_{2}+C k_{3}$ as constants, and numerical simulation result as shown in figure 1 .

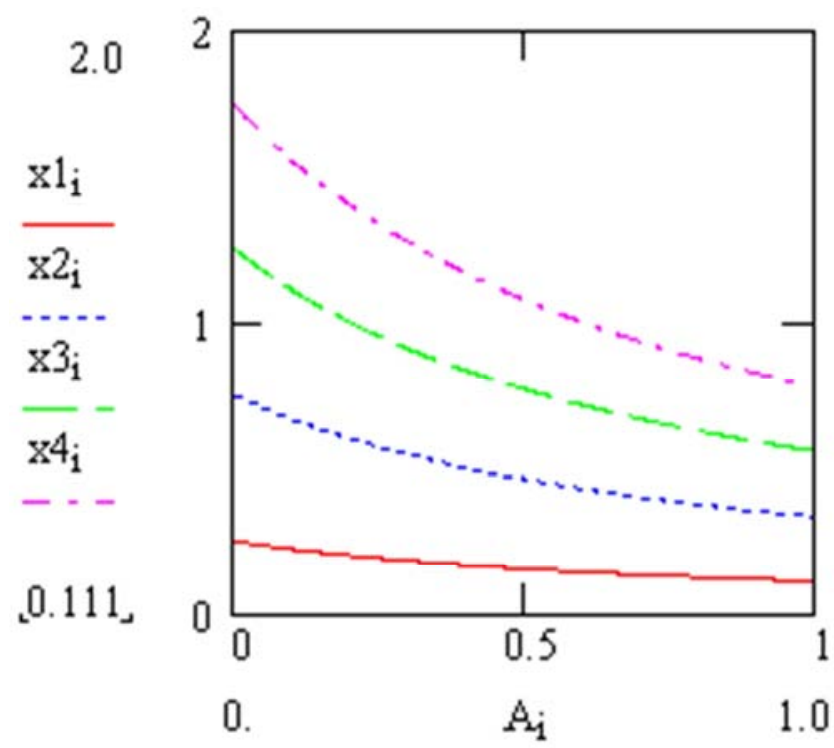

Figure 1. The relationship between the number of daytime population $x$ and the travel intensity of employed population $A$.

Where the solid line $\left(x_{1}\right)$, dot line $\left(x_{2}\right)$, dash line $\left(x_{3}\right)$ and dash dot line $\left(x_{4}\right)$ are corresponding to four conditions of $y_{m}$ taking the number of $0.1,0.3,0.5$ and 0.7 , respectively. $k_{1}$ taking the number of $0.5, B k_{2}+C k_{3}$ taking the number of 0.4 .

Figure 1 shows when $y_{m}, k_{1}$ and $B k_{2}+C k_{3}$ are constant, the relationship between the number of daytime population $x$ and the travel intensity of employed population $A$. Abscissa is the travel intensity of employed population $A$ and $A>0$, the ordinate indicates the changes of the urban daytime population as a result of changes in $A$, and the value ranges of $A$ is from $1 \%$ to $100 \%$. With the increase of $A, x$ gradually becomes smaller. Thus decrease the travel intensity of employed population who commuting into city which could increase the capacity of urban daytime population.

(2) Fix the road area, the relationship between the number of daytime population $x$ and the proportion of residential population $k_{3}$

In the urban daytime population, the larger the number of residential population who living in urban, the greater the urban space occupied by their residence and passage, and then reduce the capacity of urban daytime population. Here, we set $y_{m}, A k_{1}+B k_{2}$ and $C$ as constants, and the numerical simulation result as show in figure 2 .

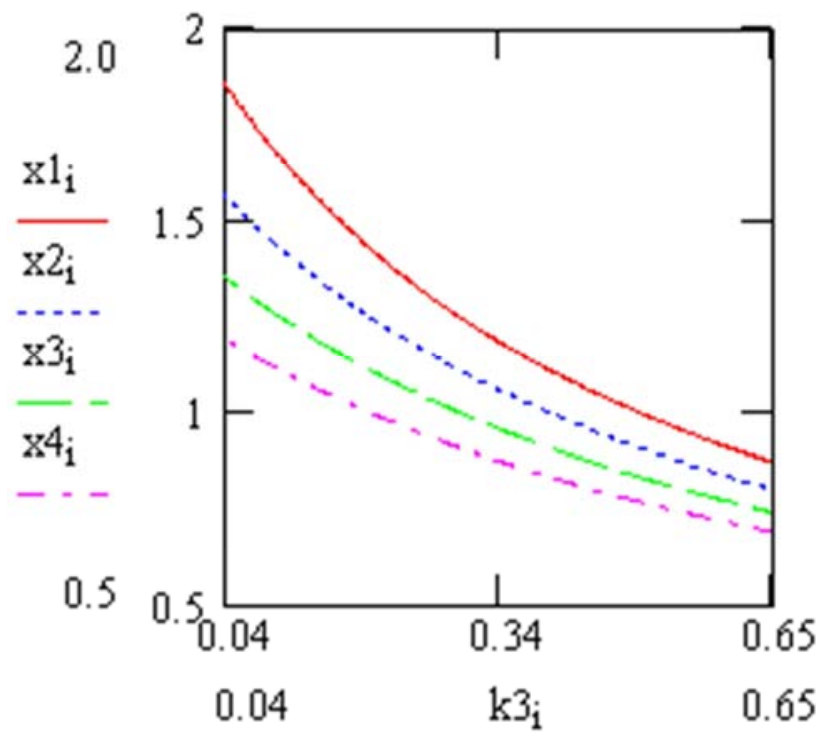

Figure 2. The relationship between the number of daytime population in cities and the proportion of the city-center residential population.

Where the solid line $\left(x_{1}\right)$, dot line $\left(x_{2}\right)$, dash line $\left(x_{3}\right)$ and dash dot line $\left(x_{4}\right)$ are corresponding to four conditions of $A k_{1}+B k_{2}$ taking the number of $0.5,0.6,0.7,0.8$, and both ${ }_{C}$ and $y_{m}$ taking the number of 1.0.

Figure 2 shows when $y_{m}, C$ and $A k_{1}+B k_{2}$ are constant, the relationship between the number of daytime population $x$ and the proportion of residential population $k_{3}$. Abscissa is the proportion of residential population and $k_{3}>0$, the ordinate indicates the changes of the urban daytime population as a result of changes in $k_{3}$, and the value ranges of $A$ is from $4 \%$ to $65 \%$ (that values are close to the population ratio of day and night population in Chiyoda District of Tokyo and Luwan District of Shanghai, respectively). With the increase of $k_{3}, x$ gradually becomes smaller. Thus decrease the proportion of residential population and increase the population ratio of day and night, which could increase the capacity of urban daytime population.

The above numerical simulation results indicate there are 
two measures to increase the capacity of urban daytime population, (i) By improving the efficiency of urban transport systems, reduce the travel intensity of commuters $A$ and $B$. In the real world, Beijing take the "Two-axis, two-belt, multi-center" space development strategy and construct Metro line 10 and line 6 through the CBD [14], which reduce the urban traffic pressure by reducing the amount of travel of commuter population. (ii) By reducing the proportion of residential population in the daytime population $k_{3}$, and the decreasing the amount of travel of residential population and reducing urban traffic pressure. For instance, the density of night population in Tokyo, New York and other international metropolitan are smaller than in Shanghai and Beijing, so the traffic pressure is relatively small [15].

\subsubsection{The Relationship Between the Total Area of Urban Road $y$ and the Maximum Road Area that a City Can Afford $y_{m}$}

$y_{m}$ is the maximum road area that a city can afford, when the traffic demands are same, the larger the maximum number of roads that a city can afford, the larger the area of road. In order to study the relationship between them, we set the through capacity of road $a\left(A k_{1}+B k_{2}+C k_{3}\right)$ as constant, and numerical simulation result as show in figure 3 .

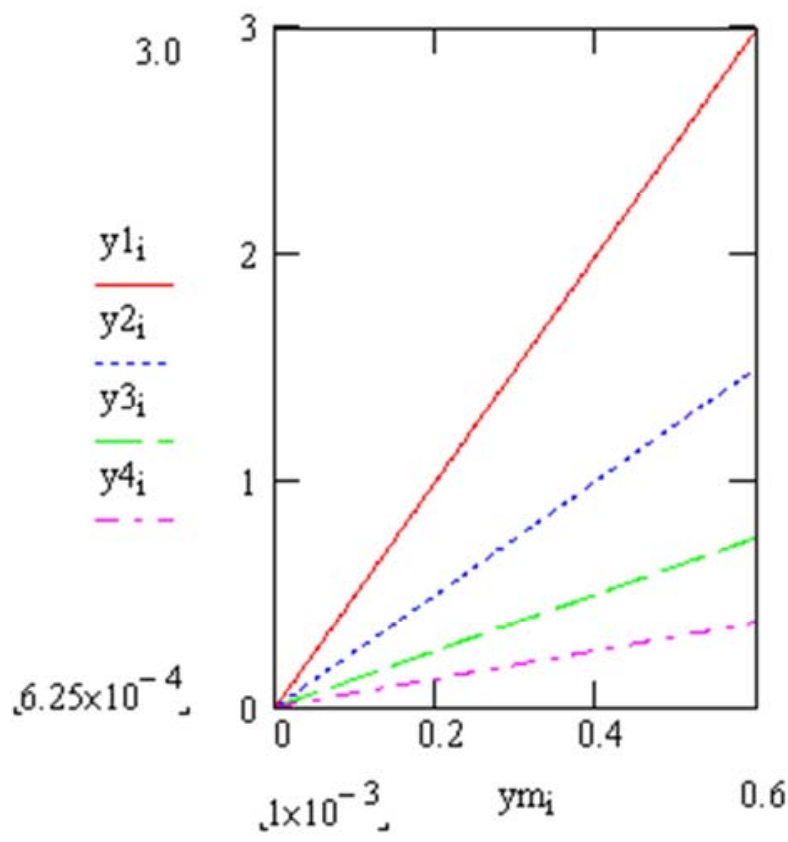

Figure 3. The relationship between the road area in a city and the maximum road area the city can bear.

Where the solid line $\left(y_{1}\right)$, dot line $\left(y_{2}\right)$, dash line $\left(y_{3}\right)$ and dash dot line $\left(y_{4}\right)$ are corresponding to four conditions of $a b$ taking the number of $0.2,0.4,0.6$ and 0.8 , respectively.

Figure 3 shows when $a\left(A k_{1}+B k_{2}+C k_{3}\right)$ is constant, the relationship between the road area $y$ and the maximum road area that a city can afford $y_{m}$. Abscissa $y_{m}$ is the maximum road area that a city can bear, the ordinate $y$ indicates the road area of a city, and the value ranges of $a\left(A k_{1}+B k_{2}+C k_{3}\right)$ is from $1 \%$ to $60 \%$. Different from figure 1 and figure 2, from figure 3 we can see that $y$ becomes larger with the increase of $y_{m}$. When the traffic demand is fixed, $y_{m}$ limit the change of $y$. In reality, it is easier to increase road areas in suburbs and rural than in city center, so the per capita road area in suburbs and rural is much larger than in city. Therefore, traffic congestion occurs mainly in the urban core area and the focus of traffic management should also be in the city core area.

\section{Some Suggestions to Urban Planning}

Through the discussion of the equilibrium state $N$, it can be obtained the relationship between the urban road area, the urban daytime population and the trip intensity, and it has some enlightenment to the urban planning.

\subsection{Urban Daytime Population Must Maintain Moderate Growth}

And should be smaller than $4 R\left(A k_{1}+B k_{2}+C k_{3}\right) / y_{m}$. If the urban daytime population growth too fast, it will be disadvantageous to the stability of the urban population and the road system. And then it may lead to urban congestion and have a negative impact on the economic and social development of city. Especially in the central areas of large cities, they should control the growth of daytime population.

\subsection{The Urban Daytime Population Is Proportional to the Road Area at Steady State}

i. Strengthen the management of urban road traffic, improve the carrying capacity of per unit road area, and then increase the number of urban daytime population.

ii. In the formulation of urban development plans, not only to consider the total population of a city, but also should take into account urban planning programs, economic development speed, industrial structure changes, CBD function enhancements and other factors that may impact on urban daytime population and its traffic demand. According to the principle of appropriate advance, rational planning of urban road land.

iii. Coordinate the relationship between road area and traffic demand. In the urban planning process, the difference of the number of daytime population, travel intensity and road capacity in different areas of the city should be considered, to avoid traffic congestion due to excessive concentration of daytime traffic demand in some areas. In order to reduce the pressure of the urban traffic that generated by the urban economic development, the urban core area with concentrated daytime population may be suitable for developing industries with small daytime traffic demand. In urban management, institutions should stagger the work time and get off time to reduce traffic flow during peak hours, to avoid traffic congestion. 


\subsection{The Growth of Urban Road Area Is Subject to the Maximum Road Area the City Can Bear}

i) In the process of urban planning, we must consider the spatial difference of urban road growth rate. Different cities and different regions of the city, the road area growth rates are different because the maximum numbers of roads they can bear are different. Widening or constructing new roads, it is more difficult for the large cities than the medium-sized cities, small towns and it is more difficult for the central areas than the suburbs.

ii) The relationship and changes between the traffic demand generated by daytime population and the urban road area affect the structure of urban traffic system and its changes. In space, different sizes of cities and different regions of the city, the structure of the transport system and its development directions are different. In large cities and the city's central areas, where are difficult to increase road area and per capita road area are relatively small, private transport with low road use efficiency should be restricted and actively develop public transport with high road utilization rate. The suburbs and small towns in large cities, due to both the daytime population and the traffic demand are small, it is difficult to develop public transport, so it should actively develop private transport with good accessibility. In time, there is an evolutionary process of urban traffic structure. With the development of the city, the probability of increasing the road area in the same area is getting smaller and smaller, the per capita road area is decreasing gradually, and the transportation system should be developed from private traffic to public transportation.

\subsection{In a Certain Road Passing Capacity, the Capacity of Urban Daytime Population Is Inversely Proportional to the Travel Intensity of Daytime Population}

i) In urban transport planning, the radial road system shortens the distance to the central area of the city, and then reduces the amount of people traveling, thus reducing road traffic pressure. Based on this reason, in the traffic development and construction planning, during the 13th five-year plan period, Beijing city constructed a number of radial rail transits.

ii) In urban spatial layout, to avoid the function of the city is too concentrated. Single-core metropolitan increase the distance of the commuter population to reach the city center, and bring great pressure to urban traffic, so this layout should be adjusted. Construct commercial and residential areas in the city center can reduce the travel amount of CBD staffs. Residential areas equipped with public facilities to meet the basic material and cultural life of the residents, which can reduce the number of trips, travel distance and then reduce the travel intensity.

iii) In the aspect of transportation mode, we draw lessons from the city layout pattern that oriented by public transportation. To attract residents to use public transport, reduce the road area occupied by residents travel, and then reduce the travel volume of daytime population. Domestic and international actual results show that the construction of public transport community can effectively reduce the traffic volume of vehicles within the community [17-19].

iv) Appropriately reduce the proportion of the residential population in the urban center area, which can increase the capacity of daytime population and ease urban traffic pressure. Strengthen the study of the urban functional areas and separation of work places and residential places, and relocate appropriately the city's residential function. Optimize the industrial structure in the central area of the city, develop the deep functions of the $\mathrm{CBD}$, protect the ecological environment and historical and cultural landscape in the central area of the city, develop the tourism resources and develop the modern service industry, so that the city center area truly become the core of the city and the power of urban expansion [20]. Concentrating a large number of high-quality daytime working population, attracting more non-working people to shopping, tourism, consulting and so on, and then reduce the proportion of the residential population in the urban center. Improve the ratio of day and night population in city center area, and ease urban traffic pressure. Some people worry that this will cause of the empty city phenomenon in the central city. In fact, at present the ratio of day and night population in the central city of China is lower than in western international metropolis, by increasing the ratio of day and night population to ease the traffic pressure in the urban center has great potential [21].

\section{Conclusions}

Based on nonlinear dynamics theory, this paper analyzes the relationship between the area of urban road and the daytime population. The author constructs the nonlinear dynamics model of the urban road and the daytime population, and calculates the equilibrium state of the dynamics system. Besides, through the discussion of the equilibrium state this paper gets the relationship between the urban road area, the urban daytime population and the trip intensity. The results show that the number of urban daytime population is proportional to the area of urban road at steady state. The author also finds that the increase of urban road area is constrained by the maximum road area of a particular area at the city. When the road area is fixed, the number of urban daytime population is inversely proportional to the travel intensity. Furthermore, this paper provides some suggestions to the urban planning.

\section{Acknowledge}

This research was supported by the project of philosophy and social science research in colleges and universities in Jiangsu province No. 2016SJB840022. 


\section{References}

[1] Kang W. Q. (2006) On the Character of Downtown Daytime Population and it's Impacts on Regional Development: A Case Study in Lu Wan District. Ph. D. Dissertation, East China Normal University, Shanghai.

[2] Shekhar S. and Xiong H. (2008) Daytime Population. Encyclopedia of Gis, 231.

[3] Yan S. Q., Xie Z. L. and Zhang H. (2005) Analysis on the Balance of the Roads and Population in the Cities Based on the Nonlinear Dynamic Model. Human Geography, 84 (4), 18-20.

[4] Zhang Y., Huang C. F. and Xu M. Z. (2016) Efficiency Research of Urban Public Transport in China Based on the Analysis of Three Stage DEA Model. Journal of Transportation Systems Engineering and Information Technology, 16 (1), 32-37.

[5] Di D., Yang D. Y. (2014) Dynamic Passenger Flow Analysis Model in Urban Public Transportation Corridor. Journal of Tongji University (Natutal Science), 42 (10), 1523-1529.

[6] Zhao P. J. and Wan H. R. (2016) Big City Traffic Characteristics and International Management Experience and Enlightenment. World Regional Studies, 25 (5), 48-57.

[7] He X. Z., Liu C. P. and Yang T. (2013) Research on the Recent Improvement Strategy and Measrue of Urban Public Transport: A Case Study of Zhenjiang City. Modern Urban Research, 12, 107-112.

[8] Xu J. P. (2016) Multi Level Measurement and Evaluation Method of the TOD. Journal of Huaqiao University (Natural Science), 37 (3), 369-374.

[9] Lin Z. S. (2003) Nonlinear Science and its Application in Earth Science. China Meteorological Press, Beijing.

[10] Lin Z. S. and Liu H. Y. (2005) Non-autonomous Population Dynamics Model for Single Species With Allee Effect Affected by Human Activities and it's Application: A Case Study of
Red-crowned Cranes Grus Japonensis. Acta Ecologica Sinica, 25 (5), 945-952.

[11] Lin Z. S. and Qi X. Z. (2005) Environmental Problems and Corresponding Counter measures About Resource Exploitation Along the Yangtze River. Resources and Environment in the Yangtze Basin, 14 (1), 24-27.

[12] Lin Z. S. (2003) The Dynamical Mechanism About Bankrupt of Petrochemical Enterprises(1). Resource Sciences, 25 (4), 2-8.

[13] Han M. A., Gu S. S. (2004) Theory and Method of Nonlinear Systems. Science Express, Beijing.

[14] Quan Y. S. (2005) Building aNew Traffic System in Beijing City. http://www.bjcbd.gov.cn/cbdplan/ 2005.12.4.16:20:53.

[15] Wang G. X. (2003) Population and Development: A Comparative Study of Shanghai and Tokyo. Fudan Journal(Social Science), 6, 10-17.

[16] The 13th Five-year's Traffic Planning in Beijing City. http://www.jiaotongjie.com/hotnews/35408.html.

[17] Li H. Y., Miao J. R. and Jiang M. (2014) Planning and Operation Management of Public Transportation in Madrid. Urban Rapid Rail Transit, 27 (1), 125-129.

[18] Zhou H. Q., Le X. H., Zhang H. X., etc. (2016) Supply Community Transit Service in Shenzhen Transit Metropolis [J]. Urban Development Studies. 23 (10), 73-78.

[19] Huang W. D. and Su X. X. (2010) Transit Oriented Community Development Based on TOD Theory-The Example of HangZhou. Urban Planning Forum, 7, 151-156.

[20] Tomoya K.. (2016) Metropolitan Rail Network and Transit Oriented Development of Tokyo. Urban Mass Transit, 11, 6-10.

[21] Yang Y. and Zhu L. B. (2001) Changes and Sustainable Development Tendency of Population Space Distribution in Shanghai. China Population, Resources and Development, 51 (11), 99-101. 